\title{
The Nexus Between Periodontal Inflammation and Dysbiosis
}

\author{
Thomas E. Van Dyke ${ }^{1 *}$, P. Mark Bartold ${ }^{2}$ and Eric C. Reynolds ${ }^{3}$ \\ ${ }^{1}$ The Forsyth Institute, Cambridge, MA, United States, ${ }^{2}$ School of Dentistry, University of Adelaide, Adelaide, SA, Australia, \\ ${ }^{3}$ Melbourne Dental School, The University of Melbourne, Melbourne, VIC, Australia
}

OPEN ACCESS

Edited by:

Philippe Saas,

INSERM U1098 Interactions

Hôte-Greffon-Tumeur \& Ingénierie

Cellulaire et Génique, France

Reviewed by:

Masae Kuboniwa,

Osaka University, Japan

Tomoki Maekawa

Niigata University, Japan

*Correspondence:

Thomas E. Van Dyke

tvandyke@forsyth.org

Specialty section:

This article was submitted to

Inflammation

a section of the journal

Frontiers in Immunology

Received: 28 January 2020 Accepted: 06 March 2020

Published: 31 March 2020

Citation:

Van Dyke TE, Bartold PM and

Reynolds EC (2020) The Nexus

Between Periodontal Inflammation

and Dysbiosis.

Front. Immunol. 11:511.

doi: 10.3389/fimmu.2020.00511
The nexus between periodontal inflammation and the polymicrobial biofilm in the gingival sulcus is critical to understanding the pathobiology of periodontitis. Both play a major role in the etiology and pathogenesis of periodontal diseases and each reinforces the other. However, this nexus is also at the center of a significant conundrum for periodontology. For all mucosal polymicrobial biofilms, the most confounding issue is the paradoxical relationship between inflammation, infection, and disease. Despite significant advances made in both periodontal microbiology and periodontal pathobiology, the issue of which comes first, the inflammatory response or the change to a dysbiotic subgingival microbiota, is still debated. In this paper, we present a model for the pathogenesis of periodontitis based on the central role of inflammation and how this modulates the polymicrobial biofilm within the context of the continuum of health, gingivitis, and periodontitis. We propose a new model termed "Inflammation-Mediated Polymicrobial-Emergence and Dysbiotic-Exacerbation" (IMPEDE), which is designed to integrate into and complement the 2017 World Workshop Classification of Periodontitis.

Keywords: periodontal inflammation, periodontal infection, periodontal pathogen, gingivitis, periodontitis

\section{INTRODUCTION}

Periodontitis is currently considered by the American Academy of Periodontology to be an inflammatory disease initiated by bacteria (1). While this represented a paradigm shift relating to the pathogenesis of periodontitis at the time, it still remains unclear how this relationship plays out during the development of periodontitis. Clearly, one cannot be there without the other. The confounding issue is the relationship between inflammation and disease; which comes first, the immune response or the change in the homeostatic integrity of the mucosal polymicrobial biofilm (2). While remarkable progress has been made in the last 10 years in both the microbiology and immunology of periodontitis, this relationship has not been clarified.

A number of questions remain to be investigated and answered. What drives the shift from the localized and contained inflammatory response of gingivitis to progressive, destructive periodontitis? When and how does the subgingival microbiome become dysbiotic? Is it a spontaneous evolution of maturing biofilms or is it driven by the changing environment mediated by the host response? What is the temporal relationship between the dysbiotic microbiome and the innate and acquired immune response? Is bacterial invasion of tissues an initiator, or consequence, of disease?

In this review, we will critically examine the evidence that addresses these questions in an effort to better understand the interface between inflammation and dysbiosis. Our understanding of the initiation of periodontitis requires reassessment. Management of disease by attempted removal of 
bacteria (debridement) is only partially effective for periodontitis and fails in high-risk individuals. Detailed consideration of the host response, genetic, and environmental factors as well as the microbiology are required. The identification of a true infectious pathogen for periodontitis has eluded researchers for decades. More recently, concepts focused on controlling the inflammation to control the polymicrobial biofilm dysbiosis have emerged for periodontitis as well as a number of other polymicrobial biofilm-based-inflammatory diseases (3-7).

\section{PATHOLOGY OF PERIODONTITIS}

The critical questions to understanding any pathological process are (1) What initiates disease, (2) What exacerbates disease, and (3) What resolves disease? The context here is periodontal disease:

\section{Initiation}

Low grade "surveillance" inflammation; that is, the presence of neutrophils in gingiva with no clinical signs of inflammation, appears to be normal homeostasis in humans. In most people, failure to disrupt the polymicrobial biofilm (plaque) accumulation at the gingival margin of teeth on a regular basis will lead to gingivitis, a destructive inflammatory lesion resulting in loss of collagen locally that is reversible upon resolution of the inflammation. Gingivitis is a chronic inflammatory lesion that in certain individuals can become destructive periodontitis. Periodontitis is distinguished from gingivitis by the destruction of supporting periodontal tissues, including periodontal ligament and alveolar bone, that is irreversible upon removal of the bacterial challenge (8). The stimulus for progression from a stable chronic gingivitis to destructive periodontitis remains elusive. To date, no specific bacterial species or antigens have been demonstrated within the tissues at this stage in the pathogenesis of periodontitis (9). Instead the initiation of destructive periodontitis has been associated with dysbiosis where the diversity, richness, and relative proportions of species in the subgingival microbiota are altered (10).

\section{Exacerbation}

We are defining this as the switch from gingivitis to periodontitis. Here, the data are limited to associations and therefore, cause and effect inferences are limited. The observations are consistent with a changing subgingival microenvironment related to chronic inflammation with a change in the composition and proportions of the bacterial species of the polymicrobial biofilm that characterizes dysbiosis. With time, there is an acquired immune response that can be either destructive or reparative in different circumstances (11). There is reasonable evidence in other systems that inflammation can cause dysbiosis (47 ), and there is reasonable evidence that dysbiosis induces inflammation (12). It is highly unlikely that either the bacteria or the host response is the sole driver of disease. However, the exact temporal relationship and contribution of each is not well-described.

\section{Resolution}

Resolution in this context refers to resolution of disease. This encompasses resolution of inflammation and return of tissue homeostasis and the re-establishment of a truly commensal plaque microbiome that is also in a homeostatic relationship with the host. This does not appear to occur spontaneously due to the heavy bacterial load that has increased species diversity that seems to be driven by the inflammatory and periodontal pocket environment. The dysbiotic subgingival plaque microbiome drives further inflammation perpetuating disease. Clinically, we have learned that this can be reversed by reduction in bacterial load that leads to reduced inflammation, or by reduction of inflammation, which prevents tissue destruction and modifies dysbiosis (13). Furthermore, this concept is supported by animal studies of experimental periodontitis where anti-inflammatory therapies not only inhibited periodontal bone resorption but also decreased the bacterial biomass and reversed dysbiosis $(14,15)$.

\section{THE INTERFACE BETWEEN INFLAMMATION AND DYSBIOSIS}

Our understanding of the relationship between inflammation and the polymicrobial biofilm was greatly enhanced by the discovery of the active pathways of resolution of inflammation and how these pathways are operative in periodontitis (16). Resolution of inflammation is regulated by Specialized Proresolving Mediators of inflammation (SPMs) comprising low molecular weight eicosanoids derived from arachidonic acid and omega-3 polyunsaturated fatty acids call lipoxins, resolvins protectins, and maresins (17). These molecules are classified as receptor agonists, not inhibitors or antagonists, that naturally resolve the inflammatory response through a feed-forward mechanism after binding to specific receptors on inflammatory cells. Since their actions are receptor mediated, their actions are specific, unlike inhibitors and antagonists. Importantly, discovery of this regulatory system opened the doors for natural manipulation of the inflammatory response in chronic inflammatory diseases. Further, it provided an avenue for the investigation of the impact of inflammation on the microbiome.

\section{Dysbiosis}

Much of the dysbiosis theory in periodontitis is an extension of gut microbiome research (18). The transition in the polymicrobial community from largely gram-positive commensal to a gram-negative enriched inflammogenic community is well-established $(19,20)$.

\section{Inflammation and Dysbiosis in Periodontitis}

The relationship of the periodontal microbiome to development of periodontitis is complex. The proposal that specific pathogens initiate dysbiosis and disease is in question due to the lack of a clear association of any putative keystone pathogen with disease initiation in humans. From microbiome analyses of plaque samples taken from healthy, gingivitis, and early periodontitis sites, we know that the bacteria associated with the 
initiation of disease are largely commensals and the "putative pathogens or pathobionts" that have been associated with disease at a later stage are very minor components of the biofilm at this early stage (20). The shift to a dysbiotic microflora appears to be in large part a function of excess and persistent inflammation and pocket formation that changes the bacterial growth environment. This was first recognized in the early 1990's as the ecological plaque hypothesis (21). In this hypothesis, the subgingival environment exerts selective pressure changing the specific microbial composition driving the change from health to disease.

The gingival microbiome associated with periodontal health is stable over time in dynamic equilibrium with the host. Gingivitis is a stable inflammatory condition and, in many ways, represents homeostasis. Excess, uncontrolled and chronic inflammation results in irreversible destruction of hard and soft tissues known as periodontitis. Disease-associated bacteria are a very small component of the subgingival microflora in health and increase significantly with the development of periodontal pockets and periodontitis $(20,22)$. In health (and in gingivitis, which is arguably the normal homeostatic condition), organisms seem to self-regulate by interspecies competition creating microbial homeostasis. With excess inflammation and pocket formation, initially by soft tissue swelling, the local environment becomes anaerobic and enriched with tissue breakdown products, plasma proteins and hemoglobin from bleeding that select for anaerobic gram-negative, proteolytic bacteria that use essential amino acids, and hemin as an energy source. Hence, overgrowth of specific subsets or consortia of microbes within the polymicrobial biofilm result from changes in the microenvironment $(21,23)$. Dysbiosis of the periodontal microbiome is clearly associated with periodontitis; however, whether dysbiosis initiates disease or is a consequence of disease initiation has not been definitively demonstrated, but it is clear that the nexus between inflammation and dysbiosis is critical $(10,24)$.

Bacteria are undoubtedly the principal cause of gingivitis, but it is the host response to those bacteria that dictates whether disease progresses (8). Overwhelming evidence has accrued to demonstrate that it is uncontrolled host inflammatory and immune responses that largely drive the tissue destruction (10).

\section{Pro-resolving Mediators and the Microbiome}

Molecular tools that directly extract and sequence cloned DNA from communities of microorganisms have opened new paths to our understanding of the relationship between human cells and the microbes that colonize the human body (25). Thousands of previously uncultured and unknown microbes have been identified. The study of the human microbiome codifies bacterial associations with inflammatory diseases and conditions including those thought to be sterile events (26). Inflammation clearly has a major impact on the microbiome in chronic inflammatory disease. For instance, pathogens that emerge can cause significant inflammatory dysregulation (27) and upregulation of systemic inflammation, as in obesity and type 2 diabetes, causes dysbiosis of the gut microbiome (28).

\section{CONVERSION OF A COMMENSAL MICROBIOTA TO AN OPPORTUNISTIC PATHOGENIC MICROBIOTA}

An important issue to consider in the pathogenesis of periodontitis is the temporal sequence of microbiome changes to periodontal inflammation. There is dysbiosis of the oral microbiome associated with periodontitis (24), but the interplay between bacteria and inflammation is just beginning to be described $(14,29)$.

Removal of plaque on the teeth reduces inflammation, but the effect is transient, and inflammation returns. Newer data suggest that susceptibility and pathogenesis of periodontitis is mediated by the host response to bacteria (1). Severe periodontitis is characterized by excess inflammation that includes oxidative stress (30) and exuberant cytokine production (31). Longitudinal studies suggest that inflammation predicts disease progression and overgrowth of pathogens in periodontitis occurs after onset of disease (32).

The SPM RvE1, when used as a topic therapeutic to treat inflammation in periodontitis prevents and reverses disease and promotes bone remodeling (23). A relevant observation is that RvE1 caused spontaneous disappearance of the periodontal pathogen, Porphyromonas gingivalis., without mechanical or antimicrobial therapy. Lee and co-workers described the temporal dynamics of inflammation-induced dysbiosis of the periodontal microbiota and the impact of RvE1 in rat experimental periodontitis (14). Global differential gene expression in periodontal tissue was determined in health, periodontitis and periodontitis treated with topical RvE1 along with $16 \mathrm{~S}$ rDNA sequencing of the associated microbiome. Topical application of $\mathrm{RvE} 1$ in rat periodontitis induced significant regeneration of lost periodontal soft tissues and bone. The shifts in the local microbiota induced by inflammation were markedly rescued by RvE1. These results illustrate two biological principles: (1) local environmental conditions impact the composition of the microbiota, and (2) the impact of inflammation on the microbiome is modifiable. These changes are not observed with inhibition of inflammation with NSAIDs or other inhibitors of inflammation (33).

Microbiome shifts induced by inflammation go beyond just overgrowth of certain species. Growth conditions also provide an environment that changes the physiology, pathogenicity, and expression of virulence factors of the polymicrobial biofilm community (10). Transcriptomic analyses performed on the microflora associated with progressive disease revealed that virulence factors are upregulated by both pathogens and health associated commensals (34). Taken together, the data suggest that the inflammatory response and the resident microbiome are linked in a bi-directional balance in health and a bi-directional imbalance in disease. These principles are not limited to oral diseases and are seen in sepsis, IBD and other diseases (35). 


\section{CAN SITE SPECIFICITY BE EXPLAINED BY INFLAMMATION MEDIATED PERIODONTAL DYSBIOSIS?}

As early as 1982, it was proposed that the specific microbiota present in deep periodontal pockets may be of significance only at a late stage of periodontal disease. The issue now is: have we made any progress in fully understanding the significance of the temporal sequence of microbial colonization, inflammation, associated tissue damage, subsequent dysbiosis followed by exacerbation of the inflammation-mediated tissue destruction? If this constant interplay between microbes and host inflammatory response is considered as a continuum, or self-sustained feedforward loop (see Figure 1), then it becomes evident that specific bacteria cannot be considered initial causal agents in the pathogenesis of periodontitis (9). More likely, the specific bacteria that have hitherto been identified as being associated with periodontitis appear because of developing disease and as late contributors. The increase in number and volume of specific bacteria is due to the inflammation and pocket formation that provides the anaerobic environment and vital nutrients, hemin, amino acids, and other growth factors via gingival crevicular fluid (36). It is important to understand the temporal and spatial sequence of events in periodontitis and pocket formation, because inflammation always precedes "periodontal pathogen" overgrowth (32). Thus, it seems probable that it is the host response and not the microbes per se that determines the eventual outcome of host parasite interactions within periodontal pockets (37). Unraveling how inflammation may drive this process is very important and it is noteworthy that induction of inflammation distant to the periodontium can induce periodontal change.

\section{Inflammation-Mediated Polymicrobial-Emergence and Dysbiotic-Exacerbation (IMPEDE) Model}

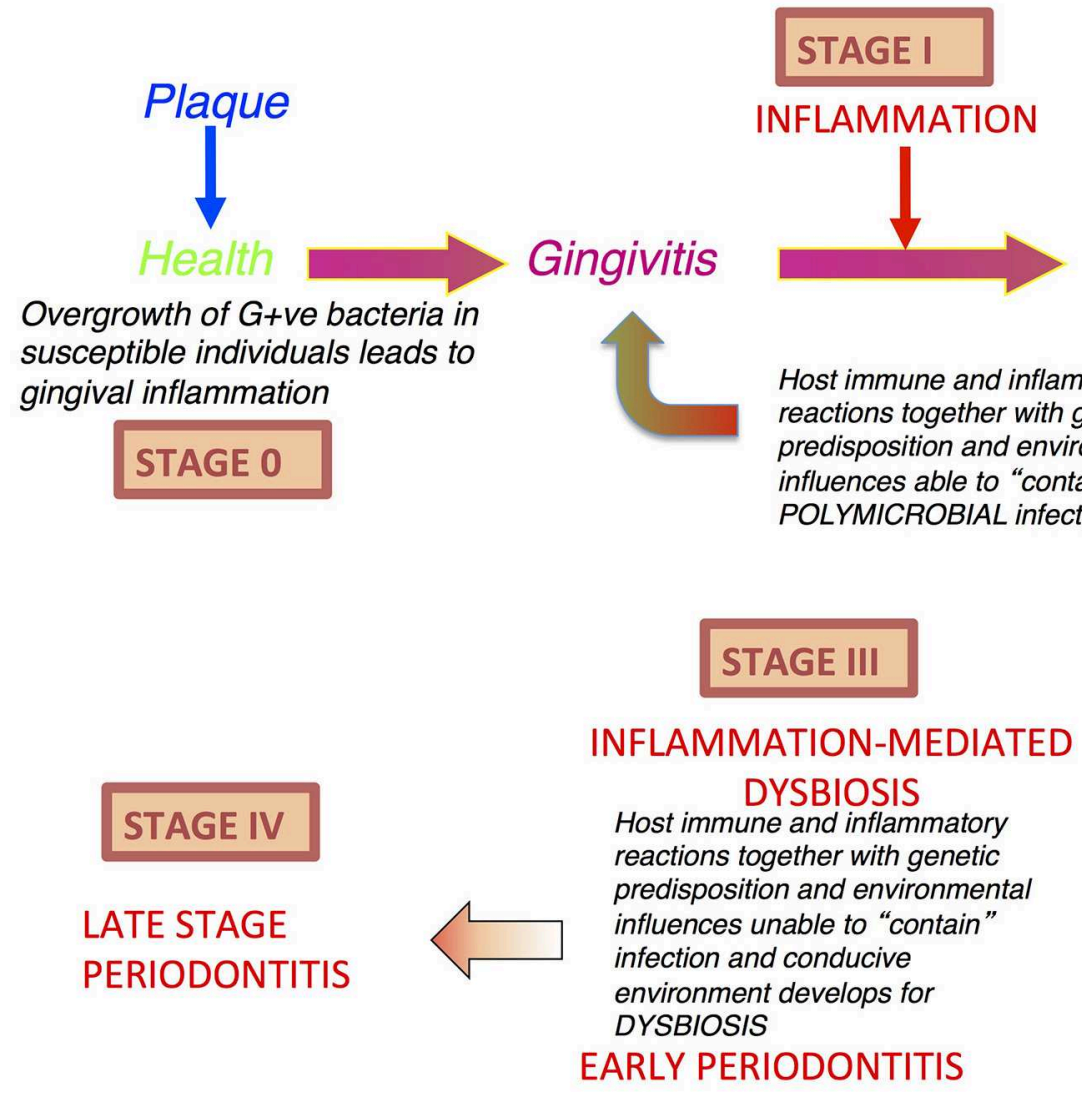

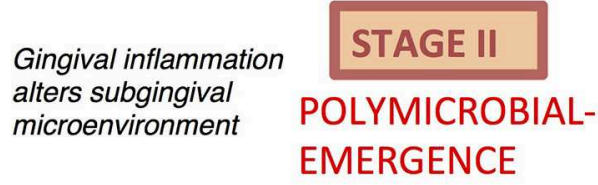
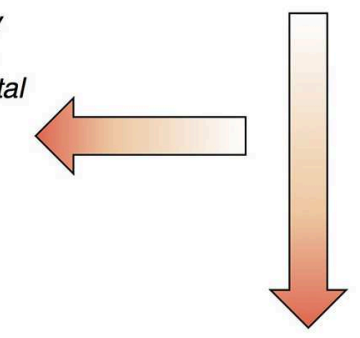

Further dysregulated host inflammation and tissue damage leading to deepening of pocket

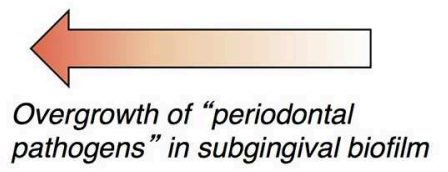

FIGURE 1 | Inflammation-Mediated Polymicrobial Emergence and Dysbiotic Exacerbation (IMPEDE) Model. A proposal to demonstrate how inflammation is a principal driver of plaque-associated periodontitis. This model recognizes 5 stages (0-IV) through which health, gingivitis, and periodontitis may develop, be contained or progress. Stage 0: periodontal health; Stage I: Gingivitis (inflammation); Stage II: Initiation/early periodontitis (Polymicrobial diversity emerges); Stage III: Inflammation mediated dysbiosis and opportunistic infection and Stage IV: Late stage periodontitis. 
With regards to pocket formation and dysbiosis, we contend that this occurs only after the initiation of chronic inflammation. The chronic inflammation allows the development of a periodontal pocket that changes the redox and nutrient environment, which increases diversity and species richness of the polymicrobial biofilm. The result is dysbiosis, which reinforces and exacerbates inflammation to initiate bone resorption. Notwithstanding this development, progression at this site is still driven by host inflammation, but now it is exacerbated by, and associated with, a dysbiotic polymicrobialhost dysregulated inflammation. Importantly, even at this stage, if inflammation can be resolved (as discussed above), then the commensal species may be able to antagonize the pathobionts such that oral homeostasis (eubiosis) can return to a treated site after debridement of the dysbiotic biofilm (23). This concept is predicated on the understanding that even with the emergence and eventual establishment of a dysbiotic subgingival polymicrobial biofilm, the commensal microbes have not been eliminated, but merely repressed until such time that the environment allows their return as the principal polymicrobial biofilm components compatible with health (38).

\section{UNIFYING CONCEPT}

In light of the above assessment of how inflammation mediates dysbiosis and associated exacerbation of periodontal damage, we propose a new unifying hypothesis called the "Inflammation-Mediated Polymicrobial-Emergence and Dysbiotic-Exacerbation" (IMPEDE) Model (Figure 1). This model is designed to complement the current Classification of Periodontal Diseases (39). In this classification, periodontitis is viewed within a continuum from health to disease through 4 stages of severity and complexity as well as extent and distribution. Within the IMPEDE model, we demonstrate how inflammation can manifest within each classification stage as a principal driver of the clinical condition (Figure 2). As for the current classification of periodontitis, the IMPEDE model recognizes 5 stages (0-4) through which health, gingivitis,

\section{IMPEDE \& Periodontal Disease Stages}

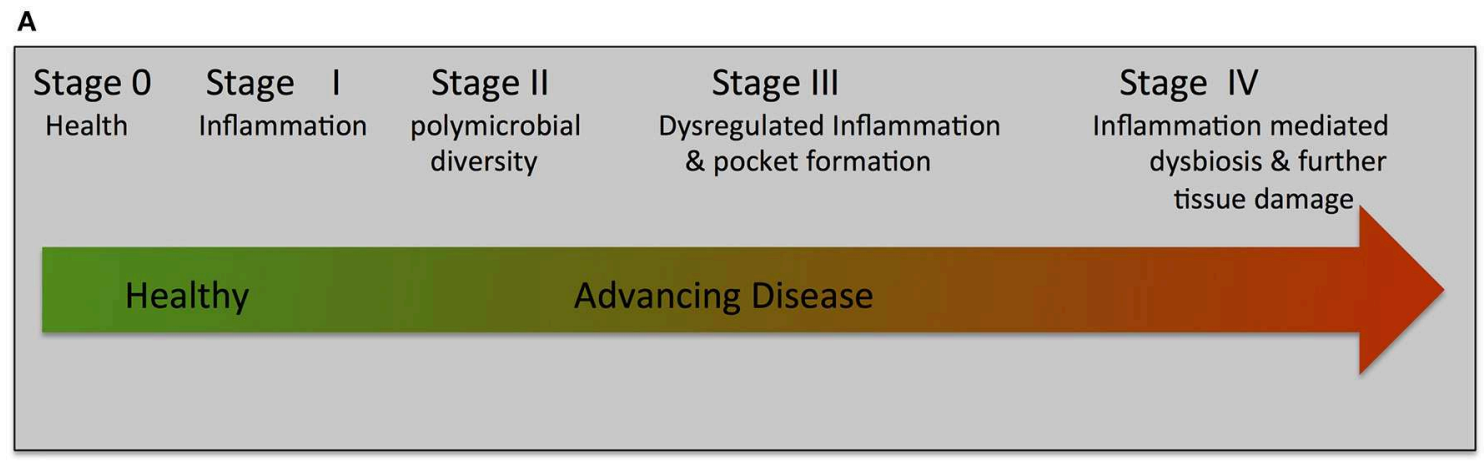

B

\begin{tabular}{|ccccc|}
\hline \multicolumn{2}{|c|}{$\begin{array}{c}\text { Inflammation Resolution-focused Treatment } \\
\text { Initiation of disease }\end{array}$} & $\begin{array}{c}\text { Inflammation Exacerbation } \\
\text { if untreated }\end{array}$ \\
No treatment & Periodontal Treatment & if treated \\
Stage 0 & Stage I & Stage II & Stage III & Stage 0 \\
& & & & \\
\end{tabular}

FIGURE 2 | IMPEDE, Periodontal Disease Classification Stages and Treatment. The IMPEDE model proposes that inflammation can manifest for each periodontitis classification stage as a principal driver of the clinical condition. In keeping within the framework of the current classification of periodontitis, the IMPEDE model identifies 5 stages (O-IV) integrating the transition from health, to gingivitis and (if untreated) ultimately to periodontitis. (A) IMPEDE stages within the new classification framework. 0 = Periodontal Health; Stage I = Gingivitis (initiation of inflammation); Stage II = Initiation/early periodontitis (Polymicrobial diversity emerges); Stage III = Advancing Periodontitis (dysregulated inflammation and pocket formation) and Stage IV = Late stage periodontitis (Inflammation mediated dysbiosis, opportunistic infection and advanced tissue destruction). (B) Inflammation-mediated polymicrobial dysbiosis and tissue damage can be exacerbated if no treatment is provided or can be driven toward resolution of inflammation and tissue repair/regeneration if treatment is provided. 
and periodontitis may develop, be contained or progress. From microbiome analyses and longitudinal clinical studies, there appear to be four different stages of bacterial transitions from health to late stage periodontitis, which is driven by inflammation, pocket formation, and bacterial composition. With health representing Stage 0 , there are four subsequent disease development phases: (1) Gingivitis (inflammation associated with an overgrowth of commensal plaque bacteria); (2) Initiation/early periodontitis (Inflammation induced polymicrobial diversity increases and dysbiosis triggered); (3) Inflammation mediated exacerbation of dysbiosis by a selfsustained feedforward loop, and (4) Late stage periodontitis characterized by a decrease in polymicrobial diversity associated with the emergence of a polymicrobial infection.

\section{Health}

Periodontal health has been clearly defined and classified and recognizes the defining feature as being absence of clinical inflammation (39). In addition, it is characterized by a plaque microbiome dominated by commensal gram-positive organisms (e.g., Streptococci, Corynebacteria, Rothia spp.) in homeostasis with the host (20). These species are antagonistic to the gramnegative species (10).

\section{Gingivitis}

Gingival inflammation in response to a non-specific accumulation of dental plaque microbiota is the defining feature of gingivitis (39). This condition is characterized by a chronic overgrowth of largely commensal organisms that in susceptible people causes inflammation and swelling of the soft tissue with early pocket formation. This results in an increase in microbial diversity in the pocket.

\section{Emergence of Polymicrobial Diversity}

It is well-established at oral and other epithelial sites that a buildup of commensal microbial biomass can trigger a switch from homeostasis (tolerance) to inflammation (40). This inflammation produces an increase in diversity of the biofilm, and this can still be associated with health at an epithelial site. Chronic inflammation (gingivitis/moderate periodontitis) can start to change the composition of the plaque with emergence of gram-negative species (21). This diverse polymicrobial biofilm is composed of disease-causing species (pathobionts) and commensal (beneficial) species (symbionts), which can be antagonistic to the pathogens. All these subgingival ecological and environmental changes are driven by the unresolved chronic inflammation, which in turn is intricately related to host susceptibility (genes and environment) and disease (tissue damage and attachment loss). As discussed above, this process can be controlled at this stage by driving resolution of inflammation allowing the commensal biofilm to return as the predominant microbiota.

\section{Dysbiosis}

In susceptible people, the chronic inflammation and anaerobic nature of the pocket results in the emergence and proliferation of certain bacterial species that may be present and an exacerbation of inflammation by the generation of a self-sustained feedforward loop to result in uncontrolled inflammation and tissue destruction $(10,41)$.

\section{OPPORTUNISTIC POLYMICROBIAL INFECTION}

There are many studies that have shown the microbial diversity of subgingival plaque increases from health to disease (gingivitis/periodontitis), which has been attributed to an increase in amount and range of nutrients provided by the exudate associated with chronic gingival inflammation (10, 42). This increase in diversity is distinct from polymicrobial infections at other sites of the body where infection can be characterized by a reduction in diversity due to increased specific pathogen abundance/competition as well as host defense mechanisms decreasing the viability/level of commensal species (43-46). However, most of the studies investigating the microbial diversity at periodontal sites have not differentiated between early/moderate disease and late stage severe periodontitis associated with deep pockets. One study by Kirst et al. (20) did differentiate between $6 \mathrm{~mm}$ pockets and those with depths $>7-8 \mathrm{~mm}$ and they showed that diversity and species richness were significantly higher in $6 \mathrm{~mm}$ pockets compared with that of the deeper pockets. The authors also showed that there was a significant increase in abundance of certain species in these deep pockets with Bacteroidetes being the most abundant. In fact, the majority ( $>50 \%)$ of species in the deeper pockets could be defined from families comprising known or suspected periodontal pathogens. This result is similar to those found by other investigators $(47,48)$ where specific sites associated with severe periodontitis were characterized by an increased abundance of known pathogens. From the Kirst et al. (20) study the authors concluded that subgingival microbial communities at diseased sites were more homogeneous than those at healthy sites suggesting a limited repertoire of species involved in disease progression. These results are consistent with a model of microbial succession in periodontitis in which disease-associated species initially invade/emerge in the healthy microbiota resulting in a diverse community comprising both health and disease-associated species. As disease (attachment loss and pocket depth) progresses, the transitional microbiota is temporally and spatially replaced by predominantly diseaseassociated species.

Recent studies on the architecture or biogeography of subgingival plaque are consistent with this model as they have revealed considerable spatial and temporal heterogeneity (4951). These studies have demonstrated considerable site specificity indicating that oral microbes are site/niche specialists and that the fine-scale positioning of a pathogen within a polymicrobial infection site can greatly alter its virulence potential. This site specificity is consistent with the known redox and nutrient gradients evident in periodontal pockets. In this context, it is significant that the periodontal pathogens Porphyromonas gingivalis and Treponema denticola are predominantly located in deep periodontal pockets at depths of more than $4 \mathrm{~mm}$ 
(52). Furthermore, these pathogens are found together with other periodontal pathogens in microcolony blooms in the biofilm surface layer adjacent to the epithelial lining at the base of deep pockets (52-56). This positioning and colocalization would advantage them in terms of release of outer membrane vesicles loaded with virulence factors into the subjacent tissue and access to the exudate from that inflamed tissue as well as being consistent with the established mutualistic symbiosis and pathogenesis displayed by these species $(55,57-59)$.

These temporal and spatial analyses of subgingival plaque help explain the disproportional effect these periodontal pathogens can have on dysregulation of the host defense to accelerate attachment loss when they appear to be only a relatively small proportion of the overall microbial biomass removed from a deep periodontal pocket (keystone pathogen hypothesis). Pathogens at the base of a periodontal pocket will be closer and therefore have more influence on subversion of the host immune response and the inflammatory processes resulting in bone resorption occurring under the pocket. These findings also help to explain why the levels of $P$. gingivalis and $T$. denticola above threshold values of around $10-15 \%$ of the microbial biomass removed from the pocket could predict imminent attachment loss in a longitudinal prospective clinical trial (60). Hence the levels of periodontal pathogens or "dysbiotic signature" of subgingival plaque may be a useful biomarker/predictor of site disease activity and imminent progression (61).

The dysbiotic signature may not only involve changes in microbial composition, but also changes in metabolomic profile $(62,63)$. Dysbiosis involves multi-directional metabolic crosstalks between subgingival micro-organisms as well as with the host such that transitions in metabolic profiles may be associated with disease progression. Recently, Sakanaka et al. (64) using saliva metabolomic data demonstrated that the metabolites cadaverine and hydrocinnamate were associated with severe periodontal inflammation. From metabolite set enrichment analysis they showed that polyamine metabolism, arginine and proline metabolism, butyric acid metabolism, and lysine degradation were distinctive metabolic signatures of severe disease.

\section{REFERENCES}

1. Van Dyke TE. Inflammation and periodontal diseases: a reappraisal. $J$ Periodontol. (2008) 79(Suppl. 8):1501-2. doi: 10.1902/jop.2008.080279

2. Dongari-Bagtzoglou A. Mucosal biofilms: challenges and future directions. Expert Rev Anti Infect Ther. (2008) 6:141-4. doi: 10.1586/14787210.6.2.141

3. Bartold PM, Van Dyke TE. Host modulation: controlling the inflammation to control the infection. Periodontol 2000. (2017) 75:317-29. doi: $10.1111 /$ prd.12169

4. Kamada N, Seo SU, Chen GY, Nunez G. Role of the gut microbiota in immunity and inflammatory disease. Nat Rev Immunol. (2013) 13:321-35. doi: $10.1038 /$ nri3430

5. Lupp C, Robertson ML, Wickham ME, Sekirov I, Champion OL, Gaynor EC, et al. Host-mediated inflammation disrupts the intestinal microbiota and
Taken together, these results indicate a process of inflammation-mediated microbial succession in periodontitis in which disease-associated species temporally and spatially emerge in a periodontal pocket resulting in a dysbiotic state to initiate periodontitis. As disease (attachment loss and pocket depth) progresses the transitional dysbiotic microbiota is further altered by an increase in abundance of predominantly disease-associated species particularly at the base of the periodontal pocket resulting in an opportunistic polymicrobial synergistic infection.

\section{CONCLUDING COMMENTS}

For many years, periodontology has experienced a conundrum with regards to the role of inflammation and microbiology in the pathogenesis of periodontitis. In this paper, we have explored the emerging evidence indicating a convergence of views with the realization of the critical nexus between the microbiology and inflammation. Current evidence suggests that the driver is the inflammation continuum and it is only at a late stage that microbial specificity (pathogenicity) starts to play a role. While this is not a new view, metagenomics, transcriptomics, proteomics, and metabolomics results in animals and humans are producing evidence to support the transition of previous theories into accepted paradigms (38). The emerging concepts presented in this paper are consistent with, and indeed complementary to, the 2017 Classification of Periodontal Diseases (39). The ramifications of these findings now allow focus on how resolving inflammation can lead to a shift in microbial composition and restoration of microbiological balance/homeostasis. In doing so, the nexus between periodontal inflammation and dysbiosis can be harnessed.

\section{AUTHOR CONTRIBUTIONS}

$\mathrm{TV}, \mathrm{PB}$, and ER contributed to conception and design of this review, and critically revised the manuscript.

\section{FUNDING}

This work was supported in part by USPHS grants DE025020 and DE025383 from NIDCR (TV).

promotes the overgrowth of enterobacteriaceae. Cell Host Microbe. (2007) 2:119-29. doi: 10.1016/j.chom.2007.06.010

6. Plotnikoff GA, Riley D. The human microbiome. Glob Adv Health Med. (2014) 3:4-5. doi 10.7453/gahmj. 2014.023

7. Zeng MY, Inohara N, Nunez G. Mechanisms of inflammation-driven bacterial dysbiosis in the gut. Mucosal Immunol. (2017) 10:18-26. doi: $10.1038 / \mathrm{mi} .2016 .75$

8. Page RC, Kornman KS. The pathogenesis of human periodontitis: an introduction. Periodontol. 2000. (1997) 14:9-11. doi: 10.1111/j.1600-0757.1997.tb00189.x

9. Bartold PM, Van Dyke TE. An appraisal of the role of specific bacteria in the initial pathogenesis of periodontitis. J Clin Periodontol. (2019) 46:6-11. doi: $10.1111 /$ jcpe. 13046 
10. Lamont RJ, Koo H, Hajishengallis G. The oral microbiota: dynamic communities and host interactions. Nat Rev Microbiol. (2018) 16:745-59. doi: 10.1038/s41579-018-0089-x

11. Cekici A, Kantarci A, Hasturk H, Van Dyke TE. Inflammatory and immune pathways in the pathogenesis of periodontal disease. Periodontol. 2000. (2014) 64:57-80. doi: 10.1111/prd.12002

12. Dutzan N, Kajikawa T, Abusleme L, Greenwell-Wild T, Zuazo CE, Ikeuchi $\mathrm{T}$, et al. A dysbiotic microbiome triggers th17 cells to mediate oral mucosal immunopathology in mice and humans. Sci Transl Med. (2018) 10:eaat0797. doi: 10.1126/scitranslmed.aat0797

13. Chen C, Hemme C, Beleno J, Shi ZJ, Ning D, Qin Y, et al. Oral microbiota of periodontal health and disease and their changes after nonsurgical periodontal therapy. ISME J. (2018) 12:1210-24. doi: 10.1038/s41396-017-0037-1

14. Lee CT, Teles R, Kantarci A, Chen T, McCafferty J, Starr JR, et al. Resolvin e1 reverses experimental periodontitis and dysbiosis. J Immunol. (2016) 197:2796-806. doi: 10.4049/jimmunol.1600859

15. Eskan MA, Jotwani R, Abe T, Chmelar J, Lim JH, Liang S, et al. The leukocyte integrin antagonist del-1 inhibits il-17-mediated inflammatory bone loss. Nat Immunol. (2012) 13:465-73. doi: 10.1038/ni.2260

16. Serhan CN, Chiang N, Van Dyke TE. Resolving inflammation: dual antiinflammatory and pro-resolution lipid mediators. Nat Rev Immunol. (2008) 8:349-61. doi: 10.1038/nri2294

17. Serhan CN, Levy BD. Resolvins in inflammation: emergence of the proresolving superfamily of mediators. J Clin Invest. (2018) 128:2657-69. doi: 10.1172/JCI97943

18. Singh VP, Proctor SD, Willing BP. Koch's postulates, microbial dysbiosis and inflammatory bowel disease. Clin Microbiol Infect. (2016) 22:594-9. doi: 10.1016/j.cmi.2016.04.018

19. Dabdoub SM, Ganesan SM, Kumar PS. Comparative metagenomics reveals taxonomically idiosyncratic yet functionally congruent communities in periodontitis. Sci Rep. (2016) 6:38993. doi: 10.1038/srep38993

20. Kirst ME, Li EC, Alfant B, Chi YY, Walker C, Magnusson I, et al. Dysbiosis and alterations in predicted functions of the subgingival microbiome in chronic periodontitis. Appl Environ Microbiol. (2015) 81:78393. doi: 10.1128/AEM.02712-14

21. Marsh PD. Microbial ecology of dental plaque and its significance in health and disease. Adv Dent Res. (1994) 8:263-71. doi: 10.1177/08959374940080022001

22. Riep B, Edesi-Neuss L, Claessen F, Skarabis H, Ehmke B, Flemmig TF, et al. Are putative periodontal pathogens reliable diagnostic markers? J Clin Microbiol. (2009) 47:1705-11. doi: 10.1128/JCM.01387-08

23. Hasturk H, Kantarci A, Goguet-Surmenian E, Blackwood A, Andry C, Serhan $\mathrm{CN}$, et al. Resolvin e1 regulates inflammation at the cellular and tissue level and restores tissue homeostasis in vivo. J Immunol. (2007) 179:7021-9. doi: 10.4049/jimmunol.179.10.7021

24. Hajishengallis G, Darveau RP, Curtis MA. The keystone-pathogen hypothesis. Nat Rev Microbiol. (2012) 10:717-25. doi: 10.1038/nrmicro2873

25. Pagani I, Liolios K, Jansson J, Chen IM, Smirnova T, Nosrat B, et al. The genomes online database (gold) v.4: status of genomic and metagenomic projects and their associated metadata. Nucleic Acids Res. (2012) 40:D571579. doi: 10.1093/nar/gkr1100

26. Rajendhran J, Shankar M, Dinakaran V, Rathinavel A, Gunasekaran P. Contrasting circulating microbiome in cardiovascular disease patients and healthy individuals. Int J Cardiol. (2013) 168:5118-20. doi: 10.1016/j.ijcard.2013.07.232

27. Helaine S, Cheverton AM, Watson KG, Faure LM, Matthews SA, Holden DW. Internalization of salmonella by macrophages induces formation of nonreplicating persisters. Science. (2014) 343:204-8. doi: $10.1126 /$ science. 1244705

28. Larsen N, Vogensen FK, van den Berg FW, Nielsen DS, Andreasen AS, Pedersen BK, et al. (2010). Gut microbiota in human adults with type 2 diabetes differs from non-diabetic adults. PLoS ONE. 5:e9085. doi: 10.1371/journal.pone.0009085

29. Sima C, Cheng Q, Rautava J, Levesque C, Sherman P, Glogauer M. Identification of quantitative trait loci influencing inflammationmediated alveolar bone loss: insights into polygenic inheritance of hostbiofilm disequilibria in periodontitis. J Periodont Res. (2016) 51:237-49. doi: $10.1111 /$ jre. 12303
30. Chapple IL, Matthews JB. The role of reactive oxygen and antioxidant species in periodontal tissue destruction. Periodontol. 2000(2007) 43:160-232. doi: $10.1111 /$ j.1600-0757.2006.00178.x

31. Graves D. Cytokines that promote periodontal tissue destruction. $J$ Periodontol. (2008) 79(Suppl. 8):1585-91. doi: 10.1902/jop.2008.080183

32. Tanner AC, Kent R Jr, Kanasi E, Lu SC, Paster BJ, Sonis ST, et al. Clinical characteristics and microbiota of progressing slight chronic periodontitis in adults. J Clin Periodontol. (2007) 34:917-30. doi: 10.1111/j.1600-051X.2007.01126.x

33. Kornman KS, Blodgett RF, Brunsvold M, Holt SC. Effects of topical applications of meclofenamic acid and ibuprofen on bone loss, subgingival microbiota and gingival pmn response in the primate macaca fascicularis. $J$ Periodont Res. (1990) 25:300-7. doi: 10.1111/j.1600-0765.1990.tb00919.x

34. Yost S, Duran-Pinedo AE, Teles R, Krishnan K, Frias-Lopez J. Functional signatures of oral dysbiosis during periodontitis progression revealed by microbial metatranscriptome analysis. Genome Med. (2015) 7:27. doi: 10.1186/s13073-015-0231-6

35. Spite M, Norling LV, Summers L, Yang R, Cooper D, Petasis NA, et al. Resolvin $\mathrm{d} 2$ is a potent regulator of leukocytes and controls microbial sepsis. Nature. (2009) 461:1287-91. doi: 10.1038/nature08541

36. Naginyte M, Do T, Meade J, Devine DA, Marsh PD. Enrichment of periodontal pathogens from the biofilms of healthy adults. Sci Rep. (2019) 9:5491. doi: 10.1038/s41598-019-41882-y

37. Mombelli A, McNabb H, Lang NP. Black-pigmenting gram-negative bacteria in periodontal disease. I topographic distribution in the human dentition. $J$ periodontal Res. (1991) 26:301-7. doi: 10.1111/j.1600-0765.1991.tb02067.x

38. Califf KJ, Schwarzberg-Lipson K, Garg N, Gibbons SM, Caporaso JG, Slots J, et al. Multi-omics analysis of periodontal pocket microbial communities pre- and posttreatment. mSystems. (2017) 2:e00016-7. doi: $10.1128 / \mathrm{mSystems.00016-17}$

39. Caton JG, Armitage G, Berglundh T, Chapple ILC, Jepsen S, Kornman KS, et al. A new classification scheme for periodontal and peri-implant diseases and conditions - introduction and key changes from the 1999 classification. $J$ Periodontol. (2018) 89(Suppl. 1):S1-S8. doi: 10.1002/JPER.18-0157

40. Belkaid Y, Harrison OJ. Homeostatic immunity and the microbiota. Immunity. (2017) 46:562-76. doi: 10.1016/j.immuni.2017.04.008

41. Hajishengallis G, Korostoff JM. Revisiting the page \& schroeder model: the good, the bad and the unknowns in the periodontal host response 40 years later. Periodontol 2000. (2017) 75:116-51. doi: 10.1111/prd. 12181

42. Griffen AL, Beall CJ, Campbell JH, Firestone ND, Kumar PS, Yang ZK, et al. Distinct and complex bacterial profiles in human periodontitis and health revealed by $16 \mathrm{~s}$ pyrosequencing. ISME J. (2012) 6:1176-85. doi: 10.1038/ismej.2011.191

43. Baishya J, Wakeman CA. Selective pressures during chronic infection drive microbial competition and cooperation. NPJ Biofilms Microbiomes. (2019) 5:16. doi: 10.1038/s41522-019-0089-2

44. Mosca A, Leclerc M, Hugot JP. Gut microbiota diversity and human diseases: should we reintroduce key predators in our ecosystem? Front Microbiol. (2016) 7:455. doi: 10.3389/fmicb.2016.00455

45. Nelson A, De Soyza A, Perry JD, Sutcliffe IC, Cummings SP. Polymicrobial challenges to koch's postulates: ecological lessons from the bacterial vaginosis and cystic fibrosis microbiomes. Innate Immun. (2012) 18:774-83. doi: $10.1177 / 1753425912439910$

46. Peters BM, Jabra-Rizk MA, O’May GA, Costerton JW, Shirtliff ME. Polymicrobial interactions: Impact on pathogenesis and human disease. Clin Microbiol Rev. (2012) 25:193-213. doi: 10.1128/CMR.00013-11

47. Hong BY, Furtado Araujo MV, Strausbaugh LD, Terzi E, Ioannidou E, Diaz PI. Microbiome profiles in periodontitis in relation to host and disease characteristics. PLoS ONE. (2015) 10:e0127077. doi: 10.1371/journal.pone.0127077

48. Ikeda E, Shiba T, Ikeda Y, Suda W, Nakasato A, Takeuchi Y, et al. Japanese subgingival microbiota in health vs disease and their roles in predicted functions associated with periodontitis. Odontology. (2019) 108:280-29. doi: 10.1007/s10266-019-00452-4

49. Mark Welch JL, Dewhirst FE, Borisy GG. Biogeography of the oral microbiome: the site-specialist hypothesis. Annu Rev Microbiol. (2019) 73:335-58. doi: 10.1146/annurev-micro-090817-062503 
50. Proctor DM, Shelef KM, Gonzalez A, Davis CL, Dethlefsen L, Burns AR, et al. Microbial biogeography and ecology of the mouth and implications for periodontal diseases. Periodontol 2000. (2020) 82:26-41. doi: $10.1111 /$ prd. 12268

51. Stacy A, McNally L, Darch SE, Brown SP, Whiteley M. The biogeography of polymicrobial infection. Nat Rev Microbiol. (2016) 14:93-105. doi: $10.1038 /$ nrmicro.2015.8

52. Kigure T, Saito A, Seida K, Yamada S, Ishihara K, Okuda K. Distribution of porphyromonas gingivalis and treponema denticola in human subgingival plaque at different periodontal pocket depths examined by immunohistochemical methods. J Periodont Res. (1995) 30:332-41. doi: 10.1111/j.1600-0765.1995.tb01284.x

53. Liu G, Chen F, Cai Y, Chen Z, Luan Q, Yu X. Measuring the subgingival microbiota in periodontitis patients: comparison of the surface layer and the underlying layers. Microbiol. Immunol. (2019) 64:99-112. doi: 10.1111/1348-0421.12759

54. Noiri Y, Li L, Yoshimura F, Ebisu S. Localization of porphyromonas gingivaliscarrying fimbriae in situ in human periodontal pockets. J Dent Res. (2004) 83:941-5. doi: 10.1177/154405910408301210

55. O'Brien-Simpson NM, Pathirana RD, Walker GD, Reynolds EC. Porphyromonas gingivalis rgpa-kgp proteinase-adhesin complexes penetrate gingival tissue and induce proinflammatory cytokines or apoptosis in a concentration-dependent manner. Infect Immun. (2009) 77:1246-61. doi: 10.1128/IAI.01038-08

56. Zijnge V, van Leeuwen MB, Degener JE, Abbas F, Thurnheer T, Gmur R, et al. Oral biofilm architecture on natural teeth. PLoS ONE. (2010) 5:e9321. doi: 10.1371/journal.pone.0009321

57. Ng HM, Slakeski N, Butler CA, Veith PD, Chen YY, Liu SW, et al. The role of treponema denticola motility in synergistic biofilm formation with porphyromonas gingivalis. Front Cell Infect Microbiol. (2019) 9:432. doi: $10.3389 /$ fcimb. 2019.00432

58. Orth RK, O'Brien-Simpson NM, Dashper SG, Reynolds EC. Synergistic virulence of porphyromonas gingivalis and treponema denticola in a murine periodontitis model. Mol Oral Microbiol. (2011) 26:229-40. doi: 10.1111/j.2041-1014.2011. 00612.x
59. Tan KH, Seers CA, Dashper SG, Mitchell HL, Pyke JS, Meuric $\mathrm{V}$, et al. Porphyromonas gingivalis and treponema denticola exhibit metabolic symbioses. PLoS Pathog. (2014) 10:e1003955. doi: 10.1371/journal.ppat.1003955

60. Byrne SJ, Dashper SG, Darby IB, Adams GG, Hoffmann B, Reynolds EC. Progression of chronic periodontitis can be predicted by the levels of Porphyromonas gingivalis and Treponema denticola in subgingival plaque. Oral Microbiol Immunol. (2009) 24:469-77. doi: 10.1111/j.1399-302X.2009.00544.x

61. Meuric V, Le Gall-David S, Boyer E, Acuna-Amador L, Martin B, Fong SB, et al. Signature of microbial dysbiosis in periodontitis. Appl. Environ. Microbiol. (2017) 83:e00462-17. doi: 10.1128/AEM.00 462-17

62. Nguyen T, Sedghi L, Ganther S, Malone E, Kamarajan P, Kapila YL. Host-microbe interactions: Profiles in the transcriptome, the proteome, and the metabolome. Periodontol 2000. (2020) 82:115-28. doi: 10.1111/prd. 12316

63. Takahashi N. Oral microbiome metabolism: From "who are they?" To “what are they doing?". J Dental Res. (2015) 94:1628-37. doi: 10.1177/0022034515606045

64. Sakanaka A, Kuboniwa M, Hashino E, Bamba T, Fukusaki E, Amano A. Distinct signatures of dental plaque metabolic byproducts dictated by periodontal inflammatory status. Sci Rep. (2017) 7:42818. doi: $10.1038 /$ srep42818

Conflict of Interest: The authors declare that the research was conducted in the absence of any commercial or financial relationships that could be construed as a potential conflict of interest.

Copyright (C) 2020 Van Dyke, Bartold and Reynolds. This is an open-access article distributed under the terms of the Creative Commons Attribution License (CC BY). The use, distribution or reproduction in other forums is permitted, provided the original author(s) and the copyright owner(s) are credited and that the original publication in this journal is cited, in accordance with accepted academic practice. No use, distribution or reproduction is permitted which does not comply with these terms. 